\title{
An Introduction to the Special Issue of QSR: "Symbolic Interactionism in Poland"
}

\author{
Krzysztof T. Konecki \\ University of Lodz, Poland
}

DOI: http://dx.doi.org/10.18778/1733-8077.16.4.01

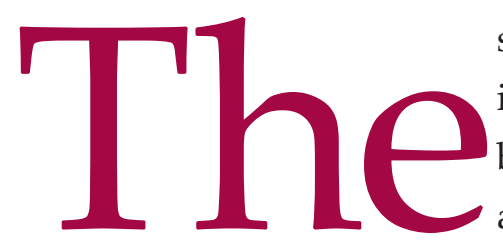

symbolic interactionist tradition initiated by the Chicago School, and later advanced by such great theoreticians and scholars as George Herbert Mead, Herbert Blumer, as well as Carl Couch, Erving Goffman, Anselm L. Strauss, Norman Denzin, or Robert Prus, to name a few, still receives recognition, as evidenced by the number of publica-

Krzysztof T. Konecki is a Full Professor at the Faculty of Economics and Sociology, University of Lodz, Poland. He is the head of the Sociology of Organization and Management Department. His interests lie in qualitative sociology, symbolic interactionism, grounded theory, the methodology of social sciences, visual sociology, communication and intercultural management, organizational culture and management, and contemplative studies. He is the editor-in-chief of the Qualitative Sociology Review, and he holds the position of the President of the Polish Sociological Association.

email address: krzysztof.konecki@uni.lodz.pl tions embedded in the perspective at hand, as well as many successful attempts to institutionalize contemporary researchers' interest in symbolic interactionism (SI). There exist several such associations like the Society for the Study of Symbolic Interaction or the European Society for the Study of Symbolic Interaction. Also, the following journals devoted to this sociological perspective are acclaimed: Symbolic Interaction, Studies in Symbolic Interaction. As to the Polish context, there is the Section of Qualitative Sociology and Symbolic Interactionism of the Polish Sociological Association and a scientific journal promoting the ideas of SI-Qualitative Sociology Review. The idea behind inviting Polish scholars to contribute to the special issue highlighting the impact of SI in Poland has been twofold-first, it is about advancing the concepts underpinning this theoretical and research perspective, second, it aims at popularizing the activity of Polish symbolic interactionists community.

Many discussions undertaking the concepts of SI and concerning issues such as the self and identity, the mind and the definition of the situation, 
taking the role of the other and socialization, deviation and constructing social order, interaction processes, communication and language, visual and material symbols, the processes of becoming, and exclusion are still timely. There are also several surfacing areas of research on interactions, such as the issue of corporeality and embodiment, as well as the awareness of the body. Moreover, new research methods aimed at capturing one's experiences, as well as lived experiences of everyday life emerge.

The authors present and discuss the research and theoretical achievements of Polish sociologists, pedagogues, social work specialists, and anthropologists whose studies are guided by SI (see the article by Konecki \& Kacperczyk). Since the Polish SI community displays great interest in doing research both inspired and framed by the concepts underpinning SI and the Chicago School perspective, presenting and discussing their achievements in the area seems to be vital in the context of grounding their research and analytic efforts. The papers dwell on theoretical and research issues (including the methodological ones) exemplified by specific theoretical achievements and/or experiences from the field, as well as respective analyses of one's or another researchers' work. This issue of QSR also offers articles presenting case studies.

As the readers will see, a lot of research employing the SI perspective that has been conducted with- in the Polish context concerns the issues of work, economy, and organizations. The identity work is investigated within the occupational contexts. The tradition of the sociology of work in Everett Hughes' terms seems to be very strong. The perspective at hand is a good fit in case of research on the meanings of work, like in the study of urban marketplaces (see the article by Marciniak), the time and space of work (see the article by Dymarczyk), emotions arising within the work context (see the article by Pawłowska), the study of freelance workers (see the article by Miller), of mobbing (see the article by Chomczyński), and regarding the work in the escort agencies in Poland (see the article by Ślęzak). There are also reflections on the influence and inspirations deriving from sociological research and methods of the Chicago School, such as uncovering the marginalized social groups (see the article by Kacperczyk). The researchers also analyze the interactive dimensions of cultural artifacts (see the article by Wiśniewski \& Bukalska), the issue of dealing with the stigma of disability (see the article by Niedbalski), and embodiment (see the article by Byczkowska-Owczarek). One can also learn how non-heteronormative mothers negotiate the meaning of motherhood in the course of interactions (see the article by Wojciechowska). The articles included in the issue exemplify diverse substantive interests of the researchers; still, each is focused on the issues regarding the process of constructing social reality, identities, space, time, and the social worlds at the time of interactions.

\section{Citation}

Konecki, Krzysztof T. 2020. “An Introduction to the Special Issue of QSR: 'Symbolic Interactionism in Poland."” Qualitative Sociology Review 16(4):6-7. Retrieved Month, Year (http://www.qualitativesociologyreview.org/ENG/archive_eng.php). DOI: http:// dx.doi.org/10.18778/1733-8077.16.4.01 